This is a post-print version. Please cite as follows and access through Elsevier here:

\author{
Andersen, E. and Maier, A. (2019) 'The attentional guidance of individual \\ colours in increasingly complex displays', Applied Ergonomics. Pergamon \\ Press, 81(November 2019). doi: 10.1016/j.apergo.2019.102885.
}

Title:

\title{
The attentional guidance of individual colours in increasingly complex displays
}

Authors:

Emil Andersen*(summadk@gmail.com), Anja Maier (amai@dtu.dk)

Affiliation:

Technical University of Denmark, DTU Management, Engineering Systems Group, Diplomvej, Kgs, Lyngby, Denmark

\section{Highlights}

- The effect of colours on attention is discussed.

- Previous research have not considered attentional effects of individual colours.

- A visual search experiment is conducted to test the attentional guidance of colours.

- Significant differences are found between individual colours.

- The effects are shown to increase with complexity of the display.

\begin{abstract}
The use of colours is a prevalent and effective tool for improving design. Understanding the effect of colours on attention is crucial for designers that wish to understand how their interfaces will be used. Previous research has consistently shown that attention is biased towards colour. However, despite previous evidence indicating that colours should be treated individually, it has thus far not been investigated whether this difference is reflected in individual effects on attention. To address this, a visual search experiment was conducted that tested the attentional guidance of six individual colours (red,blue, green, yellow, orange, purple) in increasingly complex displays. Results showed that the individual colours differed significantly in their level of guidance of attention, and that these differences increased as the visual complexity of the display increased. Implications for visual design and future research on applying colour in visual attention research and design are discussed.
\end{abstract}

Keywords

Colour; Attention; Design; Visual Interfaces; Load

Citation: Andersen, E. and Maier, A. (2019) 'The attentional guidance of individual colours in increasingly complex displays’, Applied Ergonomics. Pergamon Press, 81(November 2019). doi: 10.1016/j.apergo.2019.102885. 


\section{Introduction}

As computer technology has progressed and become more popular, the prevalence and importance of graphical interfaces, too, has increased. This tendency includes computer interfaces for use in situations such as medicine, military and nuclear power plants, where improper action may have dramatic consequences. For example, improper design of the software used by Hawaii Emergency Management Agency has been identified as possibly contributing to an operator falsely transmitting a warning about an impending missile strike to the Hawaiian population (Federal Communications Comission, 2018). A common design requirement for proper interface design is that users must be able to find specific, task-relevant objects amongst task-irrelevant objects. To achieve this, designers commonly and effectively (Vazquez, Gevers, Lucassen, van de Weijer and Baldrich, 2010) use colour to separate and group objects in visual design. For example, Starke and Barber (2018) showed that user interfaces with colour lead to faster (and equally accurate) information foraging in a complex credit card fraud detection task. Similarly, previous research on colour in applied contexts has informed guidelines for ergonomic visual design with regards to how to match object size and colour (Poulton, 1975), improve legibility through colour use for text presented on CRT (ISO 9241-306:2018, 2018; Matthews, 1987) and LCD screens (Humar et al., 2014), optimise maps through correct colour use (Francis et al., 2010; Shive and Francis, 2013), and for optimising colour background-icon combinations (Huang et al., 2008).

The importance of colour in visual design is corroborated by its importance in experimental psychology, where colour has been shown to reliably bias our attention, meaning that search is facilitated for coloured targets and that attention is more easily captured by coloured distractors (Biggs et al., 2015; Eimer and Grubert, 2014; Eimer and Kiss, 2010; Folk et al., 1994; Grubert and Eimer, 2015; Muhl-Richardson et al., 2018; Müller et al., 2009; Nordfang et al., 2013; Theeuwes, 1994; Wolfe and Horowitz, 2017, 2004). Notably, whereas the aforementioned applied studies mostly consider how visual designs of various colour combinations perform, the experimental studies of colour and attention mostly consider colour as a group (i.e. does 'colour' capture attention). The resulting research has thus aided our knowledge on combinations of colour and the attentional effects of colour as a group. However, little research has been performed on the relation between attention and specific colours. In this paper, we expand our previous discussion and findings (Andersen and Maier, 2017) on why individual colours should be expected to have distinct properties in attentional guidance and capture. We test them using an experimental design that combines the rigorous methods of experimental psychologists with the expected complexity of a realistic visual design problem.

\section{Background}

\subsection{Visual design and complex displays}

As argued by Zacks and Tversky (2003), the use of top-down principles can be greatly beneficial for design of visual interfaces. By applying knowledge acquired through studies of human behaviour in design, predictions can be made that provide insights for how to better (or optimally) create visual interfaces. Examples of such insights range from websites for everyday usability (Tsai et al., 2008), maps (Francis et al., 2010; Shive and Francis, 2013), graphs (Ratwani et al., 2008) or advertising and branding (Ko, 2017; Page et al., 2012; Resnick and Albert, 2016), to the design of nuclear control room interfaces (Braseth and Øritsland, 2013; Lau et al., 2008; Van Laar and Deshe, 2002, 2007) and hazard information systems (Miran et al., 2017). In the same vein, design guidelines based on insights from attentional theory has shown promise for improving performance of users of complex visual interfaces (McCarley and Steelman, 2013): 
Understanding the attentional system is particularly important for the design of complex visual interfaces that contain large amounts of information of which only a fraction is relevant for the user at any given time. As the foundational study by Treisman and Gelade (1980) showed, search in these situations depends on the similarity of the objects: If objects are similar, only a few items can be considered at a given moment, resulting in slow, serial search. However, if the object is distinguished from other objects by a feature that can guide attentional allocation, search will be facilitated by a pre-attentive evaluation of the objects. This distinction is corroborated by later accounts, such as the Theory of Visual Attention (Bundesen, 1990; Bundesen and Habekost, 2008), Neural Theory of Visual Attention (Bundesen et al., 2005) and Guided Search (Wolfe, 2007; Wolfe et al., 1989). Therefore, knowing which features guide and capture attention allows designers to create visual interfaces that are easy and intuitive to use, through, e.g., optimal use of colour highlighting (McDougald and Wogalter, 2014) or visual layering (Van Laar and Deshe, 2002, 2007). As outlined above, an often-used and wellestablished guiding factor has been found in colour (for a list of other potential candidates see Wolfe and Horowitz, 2004, 2017).

\subsection{Attentional guidance of colour}

A large body of work has shown that colour as a feature will capture attention if it is a distractor (e.g. Snowden, 2002; Theeuwes, 1992, 1994) or guide attention if it is a target (Biggs et al., 2015; Eimer and Grubert, 2014; Eimer and Kiss, 2010; Folk et al., 1994; Grubert and Eimer, 2015; Müller et al., 2009; Nordfang et al., 2013; Wolfe, 2007; Wolfe et al., 1989; Wolfe and Horowitz, 2004, 2017). For example, Theeuwes (1992) asked participants to search for lines embedded in a green circle amongst green squares, or green squares with a singleton red square. He found that the singleton red distractor slowed reaction time, thereby showing that attention had been captured by the distracting colour object. This finding was corroborated by Nordfang et al. (2013), who further found that the attentional capture of a colour singleton is amplified if it is a target. Recently, Biggs et al. (2015) expanded this finding, showing that the guiding of colour depends on the visual load of a display. By integrating the aforementioned Guided Search account with Load Theory (Lavie, 2010; Lavie et al., 2004; Lavie and Tsal, 1994), Biggs et al. (2015) showed that colour only guided search under high visual load. However, while these studies provide evidence that colour guides and captures attention (to varying degree, depending on the context), they have in common that they treat colour as a group, rather than investigating the effect on attention of specific colours. However, in applied contexts, colours are seldom treated as a group. Instead specific individual colours are used, for example, to comply with design choices or for colour contrast guidelines. If the attentional effects of colours differ for specific colours, colours should be considered individually for design to avoid unintended bias in navigation. While international standards and recommendations exist for measuring and optimising colour usage with regards to legibility, fidelity, and amount used (ISO 9241-306:2018, 2018), to our knowledge, no such recommendations have been made thus far for the attentional capture and guidance of specific colours. As discussed below, however, there are several reasons why the specific colours may affect attention differently.

\subsection{Reasons for individual effects of colour}

The retinas of our eyes contain a myriad of two types of photoreceptive cells commonly referred to as rods and cones. Of these, rods are sensitive to white and black, whereas cones are primarily sensitive to one of red, blue or green. All other colours are made through combinations of responses from these cells (Gazzaniga et al., 2009; Ware, 2008). Individual colours are thus treated separately at the cellular level, even before the colour signal reaches consciousness. From a cultural viewpoint, the separation of colours into primary and secondary (based on the properties of pigments) found in art (Harkness, 2006), is widely distributed and used in design

and the arts. Given these micro and macro separations of colours, it could be expected that 
individual colours are treated differently as well. Indeed, individual colours have been shown extensively to be associated with individual emotional values in subjective rating experiments: Red is most frequently associated with exciting, defending and defying/hostile moods, blue with soothing and secure, yellow and orange with stimulating, cheerful and exciting moods, green with calm and soothing and purple with dignified and stately moods (Aslam, 2006; Lee et al., 2015; Murray and Deabler, 1957; Na and Suk, 2014; Schaie, 1961b, 1961a; Wexner, 1954). Given that previous research has shown that emotional stimuli are related with higher attentional priority (Brosch and Sharma, 2005; Hodsoll et al., 2011; Huang et al., 2008; Koster et al., 2004; Ohman et al., 2001; Öhman et al., 2001) it could be extended that individual colours may have different effects on the attention system based on their emotional valence.

\subsection{Hypotheses: testing the effect of individual colour on attentional guidance and capture}

Using a visual search experiment wherein participants searched for coloured targets amongst other coloured targets, we tested Hypothesis 1:

Hypothesis 1. Individual colours have different effects on attention. The search displayed was created to be both highly controlled, thereby mimicking the studies on attention that forms much of the theoretical basis for the experiment, and complex, thereby better mimicking realworld displays. In order to investigate the primary-secondary hierarchies, the colours used were red, green, blue, yellow, orange and purple.

Furthermore, based on the findings of Biggs et al. (2015), we tested hypothesis 2:

Hypothesis 2. The effect of individual colours on attention increases as the complexity (or visual load) increases.

\section{Methods}

\subsection{Participants}

17 students were recruited through physical e-mail sign-up sheets. Participants were included if they were between 18 and 30 years old, had normal or corrected to normal eye-sight and did not suffer from any disorders that affect the attention system, such as ADHD.

Table 1 Colour codes, contrast scores and luminance scores $(\mathrm{cd} / \mathrm{m} 2)$.

\begin{tabular}{|l|l|l|l|l|l|}
\hline Colour & R & G & B & Contrast & Luminence \\
\hline Red & 255 & 0 & 0 & 4 & 29 \\
\hline Yellow & 255 & 255 & 0 & 1.1 & 106 \\
\hline Green & 0 & 176 & 80 & 2.9 & 33 \\
\hline Blue & 0 & 112 & 192 & 5.1 & 15 \\
\hline Orange & 255 & 192 & 0 & 1.6 & 66 \\
\hline Purple & 112 & 48 & 160 & 8 & 7 \\
\hline
\end{tabular}

\subsection{Apparatus}

The experiments were conducted on a laptop computer using the EPrime 2.0 software for Windows. The experiment was displayed on an 18" monitor and participants responded via an externally connected keyboard. The experiment was conducted in small, well-lit room. Upon arrival participants were greeted and asked to fill out a compliance form to verify that they complied with the inclusion criterion, and to give permission for the data to be used in 
publication. Participants were seated $\sim 60 \mathrm{~cm}$ away from the screen and with their eyes in line with the centre of the screen.

\subsection{Stimuli}

All stimuli displays were newly created for the experiment using MS PowerPoint and E-Prime 2.0 for Windows. A total of 360 target-present displays (2/3) and 180 target-absent displays (1/3) were created using six colours: Red, blue, green, yellow, orange and purple. The colour codes, contrast scores and luminance measures for each colour are shown in Table 1. The colours are shown in Fig. 1. Each display contained 20 coloured circles measuring $1.9^{\circ}$ visual angle in diameter. The target-present displays were constructed so that each colour appeared as a target with all other colour combinations as distractors and so that the target appeared both in the inner and outer circle of the display. The distractors consisted of circles of one, two, three or four different colours and were balanced so that each of the distracting colours was approximately equally represented. The locations of both targets and specific distractor colours were randomly assigned using MS Excel's = RANDOM function. Target-absent trials were included to ensure that participants had to scan the display thoroughly before responding. They were created in the same manner as target-present displays (albeit with no target).

\subsection{Procedure}

The experiment consisted of a training block ( 15 trials) and experiment block ( 540 trials). Each trial proceeded as follows: First, a screen appeared for $1500 \mathrm{~ms}$, which instructed the participant which target to search for in the following task. Second, a fixation cross appeared in the middle of the screen for $1000 \mathrm{~ms}$.

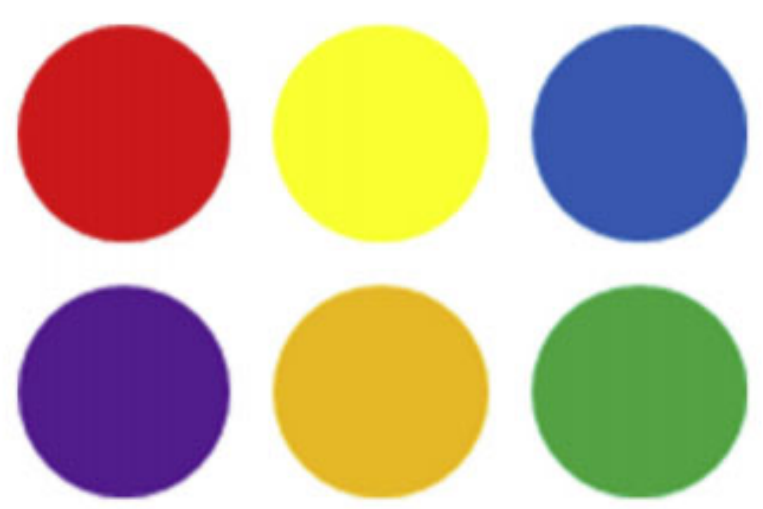

Fig. 1. The colours used in the experiment. See the online article for a coloured version of this figure. (For interpretation of the references to colour in this figure legend, the reader is referred to the Web version of this article.)

Third, the search display appeared until the participant responded with the left keyboard arrowkey if the target was present, or the right keyboard arrow-key if the target was absent. Fourth and finally, a review screen appeared which showed the participants their reaction time for the specific trial as well as their accuracy across all trials, and an instruction that the next trial could be started using the keyboard's spacebar. Fig. 2 shows the experiment procedure. 


\subsection{Analysis}

Target-absent and incorrect response trials were excluded from the dataset prior to analysis. Results were analysed using a $6 \times 4$ factorial repeated measures ANOVA model. The dependent variable was Reaction Time (RT). The independent variables were target or distractor colour (6 levels, one for each colour) and number of distractor colours (4 levels, 1 to 4 distractor colours). Subject was included as a random factor to remove inter-subject variability. Gender was tested as a co-variate given previous associations with attention control and colour perception (Harter et al., 1989; Naglieri and Rojahn, 2001), but removed from the final analysis as it did not significantly predict Reaction Time. While using parametric tests can sometimes be problematic in studies using smaller sample sizes, the assumptions of the ANOVA model were fulfilled for the present experiment after correcting for violations for the assumption of sphericity for the main effect of target colour. Analysis was conducted using base R (R Core Team, 2017) and the ezANOVA (Lawrence, 2016), ggplot2 (Wickham et al., 2018) and tidyr (Wickham, Henry, \& RStudio, 2019) packages for R.

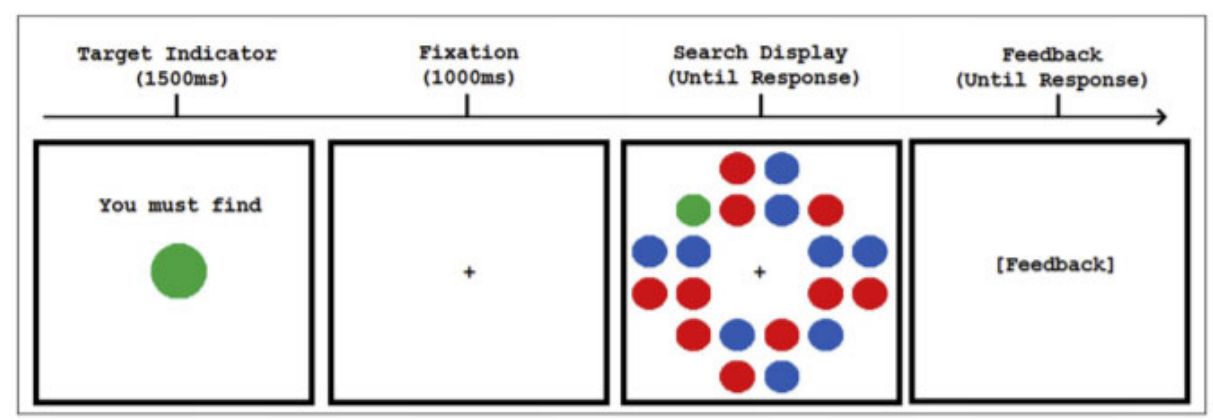

Fig. 2. The experiment procedure. See the online article for a coloured version of this figure.

Fig. 2. The experiment procedure. See the online article for a coloured version of this figure

\section{Results}

\subsection{Difference between specific target colours}

Mauchly's test indicated that the assumption of sphericity had been violated for the main effect of target colour $(\mathrm{W}=0.152, \mathrm{p}=0.02)$. Therefore degrees of freedom for the test of the effect of target colour were corrected using Greenhouse-Geisser estimates. There was a small to medium significant difference between individual target colours (F5, $80=22.91, \mathrm{p}<0.001, \eta 2=0.10)$ and a small significant difference between number of distractor colours (F3, 48 $=27.91, p<0.001, \eta 2$ $=0.06)$. There was a small significant interaction effect $(F 15,240=1.79, p=0.02, \eta 2=0.02)$, indicating a difference in search speed for individual target colours as a function of the number of distractor colours. Post hoc one way ANOVAs showed that the interaction effect was caused by a larger difference between target colours at larger amounts of distractor colours: There was a small significant difference at one distractor colour $(\mathrm{F} 5,80=4.64, \mathrm{p}<0.001, \eta 2=0.06)$, a medium significant difference at two distractor colours ( F5, $80=10.91, \mathrm{p}<0.001, \eta 2=0.13)$, and slightly larger medium significant differences at three $(\mathrm{F} 5,80=11.67, \mathrm{p}<0.001, \eta 2=0.14)$ and four $(\mathrm{F} 5$, $80=9.97, \mathrm{p}<0.001, \eta 2=0.14$ ) distractor colours (see Fig. 3). 


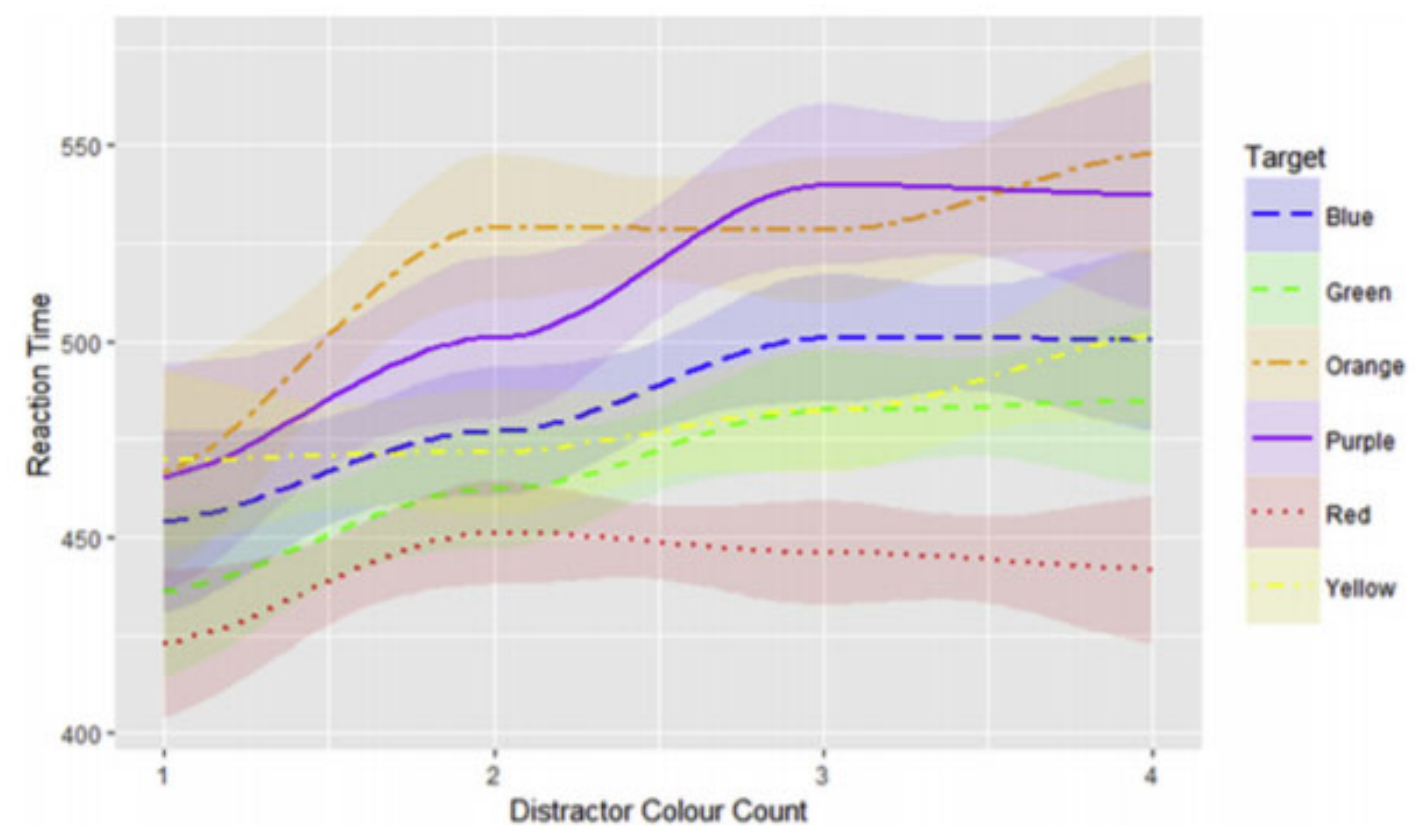

Fig. 3. The interaction between target colour and amount of different distractor colours. Error bars indicate $95 \%$ confidence intervals. See the online article for a coloured version of this graph. (For interpretation of the references to colour in this figure legend, the reader is referred to the Web version of this article.)

\subsection{Difference between individual distractor colours}

There was a small significant difference between individual distractor colours $(\mathrm{F}=5.64, \mathrm{p}<$ $0.001, \eta 2=0.06)$ and a small significant effect of distractor count $(\mathrm{F}=44.63, \mathrm{p}<0.001, \eta 2=$ 0.07). There was no significant interaction effect $(F=1.28, p=0.20, \eta 2=0.004)$, indicating that the difference in search speed for individual distractor colours did not increase as the number of distractor colours increased. Post-hoc tests revealed that red slowed search significantly more than blue $(\mathrm{p}=0.016)$, orange $(\mathrm{p}<0.001)$ and purple $(\mathrm{p}<0.001)$, while yellow slowed search significantly more than orange $(p=0.025)$ and purple $(p<0.01)$. Finally, green slowed search significantly more than purple $(\mathrm{p}=0.019)$.

\section{Discussion}

This paper investigated the hypothesis that individual colours would guide attention differently. Furthermore, based on findings by Biggs et al. (2015), the hypothesis was tested that any attentional guidance would increase as the complexity of the display increased. Our results support the hypothesis of a difference between individual colours, but show a three-way grouping: 1) red is consistently found the fastest, 2) blue, green and yellow are found slower than red, but 3) faster than orange and purple, which are found equally fast. Furthermore, our results support the hypothesis that differences in search times between individual colours increase as the complexity of the screen, induced through a higher number of differently coloured distractors, is increased. Finally, our data shows that the differences in attentional guidance are also reflected in increased attentional capture of the colours, with red, yellow and green slowing search significantly when present. 
As discussed in the introduction, several reasons could be outlined as to why there may be individual differences between colours. Our results are consistent with most accounts with regards to the colour red: red is specialized for on the cellular level, is considered a primary colour in design and art conventions and is frequently associated with threat and danger. However, for the remaining colours, neither of these three explanations completely explain the data: Explaining based on the artistic convention (i.e. red-blue-yellow is considered primary and green-orangepurple is considered secondary), is inconsistent with green being found equally fast as yellow and blue, and with red being found the fastest. Explaining based on the biology of our eyes is inconsistent with yellow being found equally fast as blue and green, and, again, is inconsistent with red being found the fastest. Explaining based on the emotional value of the colours is inconsistent with regards to how colours are grouped, as colours that are similar in their emotional meaning do not capture the same amount of attention (e.g. orange and yellow). Furthermore, the results show that red was particularly efficient at facilitating search, to the extent that search for red objects was not affected by increased complexity. It thus seemed that, unlike other colours, participants were always able to perform a parallel search for the colour red at all levels of complexity. In sum, our results show a three-group structure as the best fit for our data: 1) red, 2) green, blue and yellow, and 3) purple and orange. Furthermore, while our data does not conclusively reject that the emotion of colours was the cause of attentional guidance, our data is particularly incompatible with this explanation, as no colours that share their emotional value were found equally fast. Nevertheless, our results indicate that colours should be considered separately with regards to their attentional guidance, and that the difference in guidance increases as complexity of the display increases.

\subsection{Implications for attentional studies on colour}

Our results may contribute to both the theoretical understanding of guidance by colour, which serves as a part of several separate accounts of attention (e.g. Bundesen, 1990; Wolfe et al., 1989), and our understanding of how colour conveys emotion.

First, while previous studies have mostly attributed the guiding and capturing effect of colour to the difference in contrast (e.g. Nordfang et al., 2013, Theeuwes, 1992, 1994), our results do not corroborate this account. For example, red, which guided attention the best had a medium contrast (4), while the two colours that guided attention the least, purple and orange, had the highest (8) and second-lowest (1.6) salience scores respectively. Our results thus corroborate those of Snowden (2002) in showing that colour can capture attention in itself in a manner that is separate from luminance or contrast. While none of the proposed accounts for why colours diverge in their attentional bias explained our results perfectly, the fact that contrast was not a valid explanation either nevertheless cements our initial proposal that something about individual colours make them have individual effects on attention.

Second, while colours have previously been established as being associated with certain emotions (Lee et al., 2015; Murray and Deabler, 1957; Na and Suk, 2014; Schaie, 1961b, 1961a; Wexner, 1954), our results indicate that this emotional conveyance is either not sufficiently strong for it to guide attention, or does not occur until a later stage of processing, rendering it unable to influence attentional allocation. Of these two accounts, we find the latter explanation more likely, given the success in reproducing colour - emotion associations across diverse groups (Aslam, 2006). However, further research is needed to elucidate this relationship. 


\subsection{Implications for visual design}

As discussed in the introduction, insights on human behaviour can inform the design of visual interfaces, which in turn may lead to improved performance of their users. Indeed, the results presented here may have several applications in the design of complex visual interfaces:

The first, most apparent application of our findings lies in using colour schemes for objects that are aligned with the importance of the objects. Based on our findings, the highest priority object could thus be coloured red, whereas the least important object could be coloured purple. Furthermore, if several objects have equal importance, our findings could likewise guide designers to choosing colours that capture attention equally. Finally, our findings show that this alignment is particularly important for the design of complex visual interfaces, as the differences in guidance by colour increased with display complexity. As introduced previously, such alignment of importance and colour of objects has previously been shown to improve graph (Ratwani et al., 2008) and map design (Francis et al., 2010; Shive and Francis, 2013), and has been suggested for improving performance in $\mathrm{x}$ ray evaluation (Stroud et al., 2011). Our findings add to these by giving a general hierarchy of the role of individual colours on attention allocation, which may be useful for all types in the design of all complex visual displays. Establishing this hierarchy to understand where attention is allocated may further be applied in the design of gazecontingent displays as a way of reducing transmission bandwidth (Loschky and McConkie, 2002) or for optimising colour highlighting such as those reported by McDougald and Wogalter (2014).

Second, our finding that it may require elaborate processing before colours convey emotion can have significant consequences for how colours are used to convey semantic knowledge about products over short durations, such as in advertising, where colour plays a central role for signalling specific traits such as environmental friendliness (Aslam, 2006; Lee et al., 2015).

\section{Conclusion}

Colours as a group have been consistently shown to guide and capture attention. However, is has thus far not been investigated whether this guidance differs for individual colours. Therefore, this paper investigated experimentally whether individual colours have different levels of guidance, and whether this guidance is contingent on the complexity of the display. The results supported both these claims, showing significant differences between colours that became larger as a function of interface complexity. The data suggested a three-group structure, with 1) red facilitating search the most, 2) green, blue and yellow the second-most, and 3) orange and purple facilitating search the least. Implications for theory of perception and application to design practice were discussed. Notably, these results could not be attributed to differences in luminance or contrast. These novel empirical findings thus suggest that ergonomics guidelines for visual design may need to be developed for individual colours, in addition to those already existing with regards to luminance and contrast.

\section{Acknowledgments}

Part of the data for this study served as the basis for a contribution to the 21 st International Conference on Engineering Design (ICED17). We thank the study participants at the DTU Technical University of Denmark for their participation and our research partners from the Halden HAMMLAB at the Institute for Energy Technology (IFE) in Norway for their continued support. The work reported in this paper is funded by the Technical University of Denmark and the OECD Halden Reactor Project. 


\section{References}

Andersen, E., Maier, A., 2017. The attentional capture of colour in visual interface Design : a controlled-environment study. Proceedings of the International Conference on Engineering Design, vol. 8. ICED, pp. 519-528.

Aslam, M.M., 2006. Are you selling the right colour? A cross-cultural review of colour as a marketing cue. J. Mark. Commun. 12 (1), 15-30. https://doi.org/10.1080/13527260500247827.

Biggs, A.T., Kreager, R.D., Davoli, C.C., 2015. Finding a link between guided search and perceptual load theory. J. Cogn. Psychol. 27 (2), 164-179.

https://doi.org/10.1080/20445911.2014.987676.

Braseth, A.O., Øritsland, T.A., 2013. Visualizing complex processes on large screen displays: design principles based on the Information Rich Design concept. Displays 34 (3), 215-222. https://doi.org/10.1016/j.displa.2013.05.002.

Brosch, T., Sharma, D., 2005. The role of fear-relevant stimuli in visual search: a comparison of phylogenetic and ontogenetic stimuli. Emotion 5 (3), 360-364. https://doi.org/10.1037/15283542.5.3.360.

Bundesen, C., 1990. A theory of visual attention. Psychol. Rev. 97 (4), 523-547. https://doi.org/10.1037/0033-295X.97.4.523.

Bundesen, C., Habekost, T., 2008. Principles of Visual Attention: Linking Mind and Brain (lt;oxdc:e). Oxford University Press.

Bundesen, C., Habekost, T., Kyllingsbæk, S., 2005. A neural theory of visual attention: bridging cognition and neurophysiology. Psychol. Rev. 112 (2), 291-328. https://doi. org/10.1037/0033295X.112.2.291.

Eimer, M., Grubert, A., 2014. The gradual emergence of spatially selective target processing in visual search: $\{$ From $\}$ feature-specific to object-based attentional control.

J. Exp. Psychol. Hum. Percept. Perform. 40 (5), 1819-1831. https://doi.org/10. 1037/a0037387.

Eimer, M., Kiss, M., 2010. Top-down search strategies determine attentional capture in visual search: behavioral and electrophysiological evidence. Atten. Percept. Psychophys. 72 (4), 951962. https://doi.org/10.3758/APP.72.4.951.

Federal Communications Comission, 2018. Presentation on Preliminary Report on Hawaii False Emergency Alert. Federal Communications Commission. Retrieved from.https://www.fcc.gov/document/presentation-preliminary-report-hawaii-falseemergencyalert.

Folk, C.L., Remington, R.W., Wright, J.H., 1994. The structure of attentional control: contingent attentional capture by apparent motion, abrupt onset, and color. J. Exp. Psychol. Hum. Percept. Perform. 20 (2), 317-329. https://doi.org/10.1037/0096-1523.20.2.317.

Francis, G., Bias, K., Shive, J., 2010. The psychological four-color mapping problem. J. Exp. Psychol. Appl. 16 (2), 109-123. https://doi.org/10.1037/a0019095.

Gazzaniga, M.S., Ivry, R.B., Mangun, G.R., 2009. Cognitive neuroscience: the biology of the mind. The Quarterly Review of Biology, vol. 84. WWNorton, pp. 3.

https://doi.org/10.1086/603482. 
Grubert, A., Eimer, M., 2015. Rapid parallel attentional target selection in single-color and multiple-color visual search. J. Exp. Psychol. Hum. Percept. Perform. 41 (1), 86-101. https://doi.org/10.1037/xhp0000019.

Harkness, N., 2006. The colour wheels of art, perception, science and physiology. Optics \& Laser Technology. https://doi.org/10.1016/j.optlastec.2005.06.010.

Harter, M.R., Miller, S.L., Price, N.J., LaLonde, M.E., Keyes, A.L., 1989. Neural processes involved in directing attention. J. Cogn. Neurosci. 1 (3), 223-237. https://doi.org/10.1162/jocn.1989.1.3.223.

Hodsoll, S., Viding, E., Lavie, N., 2011. Attentional capture by irrelevant emotional distractor faces. Emotion 11 (2), 346-353. https://doi.org/10.1037/a0022771.

Huang, Y.M., Baddeley, A., Young, A.W., 2008. Attentional capture by emotional stimuli is modulated by semantic processing. J. Exp. Psychol. Hum. Percept. Perform. 34 (2), 328-339. https://doi.org/10.1037/0096-1523.34.2.328.

Humar, I., Gradisar, M., Turk, T., Erjavec, J., 2014. The impact of color combinations on the legibility of text presented on LCDs. Appl. Ergon. 45 (6), 1510-1517.

https://doi.org/10.1016/j.apergo.2014.04.013.

ISO 9241-306:2018, 2018. Ergonomics of Human-System Interaction - Part 306: Field Assessment Methods for Electronic Visual Displays. Retrieved from.

https://sd.ds.dk/Viewer/Standard?ProjectNr=M310685\&Status=60.60\&VariantID $=\& P a g e=0$.

Ko, Y.H., 2017. The effects of luminance contrast, colour combinations, font, and search time on brand icon legibility. Appl. Ergon. 65, 33-40. https://doi.org/10.1016/j.apergo.2017.05.015.

Koster, E.H.W., Crombez, G., Van Damme, S., Verschuere, B., De Houwer, J., 2004. Does imminent threat capture and hold attention? Emotion 4 (3), 312-317. https://doi.org/10.1037/1528-3542.4.3.312.

Lau, N., Veland, Ø., Kwok, J., Jamieson, G.A., Burns, C.M., Braseth, A.O., Welch, R., 2008. Ecological interface design in the nuclear domain: an application to the secondary subsystems of a boiling water reactor plant simulator. IEEE Trans. Nucl. Sci. 55 (6), 3579-3596.

https://doi.org/10.1109/TNS.2008.2005979.

Lavie, N., 2010. Attention, distraction, and cognitive control under load. Curr. Dir. Psychol. Sci. 19 (3), 143-148. https://doi.org/10.1177/0963721410370295.

Lavie, N., Hirst, A., De Fockert, J.W., Viding, E., 2004. Load theory of selective attention and cognitive control. J. Exp. Psychol. Gen. 133 (3), 339-354. https://doi.org/10.1037/00963445.133.3.339.

Lavie, N., Tsal, Y., 1994. Perceptual load as a major determinant of the locus of selection in visual attention. Percept. Psychophys. 56 (2), 183-197. https://doi.org/10.3758/BF03213897.

Lawrence, M.A., 2016. November). Ez: Easy Analysis and Visualization of Factorial Experiments. Retrieved from. https://cran.r-project.org/package=ez. Lee, J., Jung, B., Chu, W., 2015. Signaling environmental altruism through design: the role of green cue prominence in hybrid cars. Int. J. Des. 9 (2), 79-91.

Loschky, L.C., McConkie, G.W., 2002. Investigating spatial vision and dynamic attentional selection using a gaze-contingent multiresolutional display. J. Exp. Psychol. Appl. 8 (2), 99-117. https://doi.org/10.1037//1076-898X.8.2.99. 
Matthews, M.L., 1987. The influence of colour on CRT reading performance and subjective comfort under operational conditions. Appl. Ergon. 18 (4), 323-328.

https://doi.org/10.1016/0003-6870(87)90140-2.

McCarley, J.S., Steelman, K.S., 2013. Visual attention and display design. In: Neuroergonomics: A Cognitive Neuroscience Approach to Human Factors and Ergonomics. Palgrave Macmillan, London, pp. 51-68. https://doi.org/10.1057/9781137316523_3.

McDougald, B.R., Wogalter, M.S., 2014. Facilitating pictorial comprehension with color highlighting. Appl. Ergon. 45 (5), 1285-1290. https://doi.org/10.1016/j.apergo.2013.05.008.

Miran, S.M., Ling, C., James, J.J., Gerard, A., Rothfusz, L., 2017. User perception and interpretation of tornado probabilistic hazard information: comparison of four graphical designs. Appl. Ergon. 65, 277-285. https://doi.org/10.1016/j.apergo.2017.06.016.

Muhl-Richardson, A., Godwin, H.J., Garner, M., Hadwin, J.A., Liversedge, S.P., Donnelly, N., 2018. Individual differences in search and monitoring for color targets in dynamic visual displays. J. Exp. Psychol. Appl. https://doi.org/10.1037/xap0000155.

Müller, H.J., Geyer, T., Zehetleitner, M., Krummenacher, J., 2009. Attentional capture by salient color singleton distractors is modulated by top-down dimensional set. J. Exp.Psychol. Hum. Percept. Perform. 35 (1), 1-16. https://doi.org/10.1037/0096-1523.35.1.1.

Murray, D.C., Deabler, H.L., 1957. Colors and mood-tones. J. Appl. Psychol. 41 (5), 279-283. https://doi.org/10.1037/h0041425.

Na, N., Suk, H.J., 2014. The emotional characteristics of white for applications of product color design. Int. J. Des. 8 (2), 61-70. https://doi.org/10.1007/978-3-319-04798-0_20.

Naglieri, J.A., Rojahn, J., 2001. Gender differences in planning, attention, simultaneous, and successive ( $\{$ PASS $\}$ ) cognitive processes and achievement. J. Educ. Psychol. 93 (2), 430-437. https://doi.org/10.1037//0022-0663.93.2.430.

Nordfang, M., Dyrholm, M., Bundesen, C., 2013. Identifying bottom-up and top-down components of attentional weight by experimental analysis and computational modeling. J. Exp. Psychol. Gen. 142 (2), 510-535. https://doi.org/10.1037/a0029631.

Ohman, A., Flykt, A., Esteves, F., 2001. Emotion drives attention_Snakes in the grass.pdf. J. Exp. Psychol. Gen. 130 (3), 466-478. https://doi.org/10.1037/AXJ96-3445.130.3.466.

Öhman, A., Lundqvist, D., Esteves, F., 2001. The face in the crowd revisited: a threat advantage with schematic stimuli. J. Personal. Soc. Psychol. 80 (3), 381-396. https://doi.org/10.1037/00223514.80.3.381.

Page, T., Thorsteinnson, G., Ha, J.G., 2012. Using colours to alter consumer behaviour and product success. Int. J. Contents 8 (1), 69-73. https://doi.org/10.5392/IJoC.2012.8.1.069.

Poulton, E.C., 1975. Colours for sizes: a recommended ergonomic colour code. Appl.Ergon. 6 (4), 231-235. https://doi.org/10.1016/0003-6870(75)90116-7.

R Core Team, 2017. R: A Language and Environment for Statistical Computing. R Foundation for Statistical Computing, Vienna, Austria Retrieved from. https://www.r-project.org/.

Ratwani, R.M., Trafton, J.G., Boehm-Davis, D.A., 2008. Thinking graphically: connecting vision and cognition during graph comprehension. J. Exp. Psychol. Appl. 14 (1), 36-49.

https://doi.org/10.1037/1076-898X.14.1.36. 
Resnick, M.L., Albert, W., 2016. The Influences of design esthetic, site relevancy and task relevancy on attention to banner advertising. Interact. Comput. 28 (5), 680-694. https://doi.org/10.1093/iwc/iwv042.

Schaie, K.W., 1961a. A $\{$ Q \}-sort study of color-mood association. J. Projective. Tech. 25 (3), 341-346. https://doi.org/10.1080/08853126.1961.10381048.

Schaie, K.W., 1961b. Scaling the association between colors and mood-tones. Am. J. Psychol. 74 (2), 266. https://doi.org/10.2307/1419412.

Shive, J., Francis, G., 2013. Choosing colors for map display icons using models of visual search. Hum. Factors 55 (2), 373-396. https://doi.org/10.1177/0018720812459341.

Snowden, R.J., 2002. Visual attention to color: parvocellular guidance of attentional resources? Psychol. Sci. 13 (2), 180-184. https://doi.org/10.1111/1467-9280.00433.

Starke, S.D., Baber, C., 2018. The effect of four user interface concepts on visual scan pattern similarity and information foraging in a complex decision making task. Appl.

Ergon. 70, 6-17. https://doi.org/10.1016/j.apergo.2018.01.010.

Stroud, M.J., Menneer, T., Cave, K.R., Donnelly, N., Rayner, K., 2011. Search for multiple targets of different colors : misguided eye movements reveal a reduction of color selectivity. Appl. Cognit. Psychol. 25 (6), 971-982. Retrieved from. http://onlinelibrary.wiley.com/doi/10.1002/acp.1790/full.

Theeuwes, J., 1992. Perceptual selectivy for color and form. Percept. Psychophys. 51 (6), 599606.

Theeuwes, J., 1994. Stimulus-driven capture and attentional set: selective search for color and visual abrupt onsets. J. Exp. Psychol. Hum. Percept. Perform. 20 (4), 799-806. https://doi.org/10.1037/0096-1523.20.4.799.

Treisman, A.M., Gelade, G., 1980. A feature-integration theory of attention. Cogn. Psychol. 12 (1), 97-136. https://doi.org/10.1016/0010-0285(80)90005-5.

Tsai, T.W., Chang, T.C., Chuang, M.C., Wang, D.M., 2008. Exploration in emotion and visual information uncertainty of websites in culture relations. Int. J. Des. 2 (2), 55-66. Retrieved from. $\mathrm{http} / / /$ search.proquest.com/openview/6f02f4f21767f784f5aecc2ac9d20634/1?pqorigsite $=$ gscholar.

Van Laar, D., Deshe, O., 2002. Evaluation of a visual layering methodology for colour coding control room displays. Appl. Ergon. 33 (4), 371-377. https://doi.org/10.1016/S00036870(01)00048-5.

Van Laar, D., Deshe, O., 2007. Color coding of control room displays: the psychocartography of visual layering effects. Hum. Factors: J. Human Factors Ergon. Soc. 49 (3), 477-490. https://doi.org/10.1518/001872007X200111.

Vazquez, E., Gevers, T., Lucassen, M., van de Weijer, J., Baldrich, R., 2010. Saliency of color image derivatives: a comparison between computational models and human perception. J. Opt. Soc. Am. A 27 (3), 613. https://doi.org/10.1364/JOSAA.27.000613.

Ware, C., 2008. Visual Thinking: for Design. Ergonomics, vol. 53 Morgan Kaufmann. https://doi.org/10.1080/00140130903458285. 
Wexner, L.B., 1954. The degree to which colours (hues) are associated with mood tones. J. Appl. Psychol. 38 (6), 432-435. https://doi.org/10.1037/h0062181.

Wickham, H., Chang, W., Henry, L., Pedersen, T.L., Takahashi, K., Wilke, C., et al., 2018. ggplot2: Create Elegant Data Visualisations Using the Grammar of Graphics. Retrieved from. https://cran.r-project.org/package=ggplot2 .

Wickham, H., Henry, L., RStudio, 2019. Tidyr: Easily Tidy Data with "Spread" and "Gather" Functions. Retrieved from. https://cran.r-project.org/package=tidyr.

Wolfe, J.M., 2007. Guided search 4.0. Integrated Models of Cognitive Systems (3), 99-120. https://doi.org/10.1007/978-94-011-5698-1_30.

Wolfe, J.M., Cave, K.R., Franzel, S.L., 1989. Guided search: an alternative to the feature integration model for visual search. J. Exp. Psychol. Hum. Percept. Perform. 15 (3), 419-433.

Wolfe, J.M., Horowitz, T.S., 2004. What attributes guide the deployment of visual attention and how do they do it? Nat. Rev. Neurosci. 5 (6), 495-501. https://doi.org/10.1038/nrn1411.

Wolfe, J.M., Horowitz, T.S., 2017. Five factors that guide attention in visual search. Nat. Human Behaviour 1 (3), 58. https://doi.org/10.1038/s41562-017-0058.

Zacks, J.M., Tversky, B., 2003. Structuring information interfaces for procedural learning. J. Exp. Psychol. Appl. 9 (2), 88-100. https://doi.org/10.1037/1076-898X.9.2.88. 